\title{
A maximum feasible subset algorithm with application to radiation therapy
}

\section{Sadegh, Payman}

\section{Published in:}

Proceedings of American Control Conference

Link to article, DOI:

10.1109/ACC.1999.782859

Publication date:

1999

Document Version

Publisher's PDF, also known as Version of record

Link back to DTU Orbit

Citation (APA):

Sadegh, P. (1999). A maximum feasible subset algorithm with application to radiation therapy. In Proceedings of American Control Conference (Vol. 1, pp. 405-408) https://doi.org/10.1109/ACC.1999.782859

\section{General rights}

Copyright and moral rights for the publications made accessible in the public portal are retained by the authors and/or other copyright owners and it is a condition of accessing publications that users recognise and abide by the legal requirements associated with these rights.

- Users may download and print one copy of any publication from the public portal for the purpose of private study or research.

- You may not further distribute the material or use it for any profit-making activity or commercial gain

- You may freely distribute the URL identifying the publication in the public portal

If you believe that this document breaches copyright please contact us providing details, and we will remove access to the work immediately and investigate your claim 


\title{
A Maximum Feasible Subset Algorithm with Application to Radiation Therapy
}

\author{
Payman Sadegh \\ Dept. of Mathematical Modeling, Tech. University of Denmark, DK-2800 Lyngby, Denmark. \\ psQimm.dtu.dk
}

\begin{abstract}
Consider a set of linear one sided or two sided inequality constraints on a real vector $X$. The problem of interest is selection of $X$ so as to maximize the number of constraints that are simultaneously satisfied, or equivalently, combinatorial selection of a maximum cardinality subset of feasible inequalities. Special classes of this problem are of interest in a variety of areas such as pattern recognition, machine learning, operations research, and medical treatment planning. This problem is generally solvable in exponential time. A heuristic polynomial time algorithm is presented in this paper. The algorithm relies on an iterative constraint removal procedure where constraints are eliminated from a set proposed by solutions to minmax linear programs. The method is illustrated by a simulated example of a linear system with double sided bounds and a case from the area of radiation therapy.
\end{abstract}

\section{Introduction}

Consider the set $\left\{X^{\top} a_{i} \mid a_{i}, X \in \mathcal{R}^{p}, i=1, \cdots, N\right\}$ where typically $N \gg p$. Assume that for each element, $X^{\top} a_{i}$, a one sided or two sided bound is given. The problem of interest is selection of $X$ so as to maximize the number of elements that simultaneously lie within their corresponding bounds. Special classes of this problem are of interest in areas such as pattern recognition [1], machine learning [2, 3], and medical treatment planning. The numerical study of the paper considers a problem from the area of medical treatment planning.

The problem may be formulated as a combinatorial optimization with the objective of selecting a maximum cardinality subset of $\left\{X^{\top} a_{i}\right\}$ that together with their corresponding bounds define a feasible system. The variable $X$ is then found as a point in the selected feasible region. This combinatorial problem is solvable only in exponential time [4]. In [1] an algorithm is proposed for a particular class of pattern recognition problems which is better than exhaustive search. The problem may alternatively be formulated as a continuous search over $X$ where the objective function is the number of elements that lie within the given bounds. The continuous formulation does not remedy the complexity of the problem since the objective function is discontinuous and typically exhibits numerous local optima. As a result, the usual gradient based algorithms are not appropriate. Techniques such as genetic algorithm may be useful but not very efficient. Other heuristic search procedures have been proposed that apply to particular classes of the above problem [2]. The problem has been studied from a linear programming infeasibility analysis point of view [5]. [6] presents an algorithm based on alternative formulation of the problem as a minimum weight cover for irreducible inconsistent subsystems (an infeasible subsystem with smallest number of elements). The approach of [6] relies on integer programming and suffers from complexity and difficulty of implementation. The approach followed in this paper is in spirit close to the work of $[5,7]$. In both approaches a polynomial time algorithm is proposed that at each iteration solves a linear program to determine candidate inequalities for removal from the set. These candidates are removed and the procedure is repeated until a feasible subset is rendered. The main difference between the approach here and the work of $[5,7]$ is in the constraint removal technique. While [7] determines removal candidates for elimination by solving an elastic linear program [8] obtained by minimizing the sum of positive violations from the bounds, the constraint removal of the present work relies on solutions to minmax linear programs. The advantage is reduced number of variables for the linear programs (solved at each iteration) and much fewer number of candidates for removal. Moreover, should there at each iteration exist a single constraint whose elimination renders a feasible subset, that single constraint will be identified by the elimination process of the algorithm.

The rest of the paper is organized as follows. The algorithm is presented in Section 2 and illustrated by two numerical examples in Section 3 which contains a 
simulated linear system with two sided bounds and a case from radiation therapy planning. Section 4 offers concluding remarks.

\section{Maximum feasible subset algorithm}

In this section, the algorithm for maximum feasible subset selection is presented. As mentioned in the introduction, the algorithm relies on iterative solutions to minmax linear programs.

Let $S$ denote $\left\{\left(a_{i}, L_{i}, U_{i}\right) \mid i=1, \cdots, N\right\}$ and consider double sided bounds of the form $L_{i} \leq X^{\top} a_{i} \leq U_{i}$ on $X$. Generalization to the case where some or all constraints are one sided is straightforward. The problem of interest is maximization (with respect to $X$ ) of $\sum_{i} I\left(L_{i} \leq X^{\top} a_{i} \leq U_{i}\right)$ where the indicator function $I(\cdot)$ is equal to 1 if its argument is true and 0 otherwise. The above optimization problem is equivalent to minimization of the sum $\sum_{i} \alpha_{i}^{q}, q \rightarrow 0^{+}$, where $\alpha_{i}=\max \left\{0, \max \left\{X^{\top} a_{i}-U_{i}, L_{i}-X^{\top} a_{i}\right\}\right\}$, ie largest positive deviation from the bounds for the $i$ 'th constraint (note that $\alpha_{i}^{0^{+}}$is zero if $\alpha_{i}=0$ and 1 otherwise). Hence, the problem may be regarded as minimizing the $0^{+}$-norm of the (deviation) sequence $\left\{\alpha_{i}\right\}$ with respect to $X$. The objective function $\sum_{i} \alpha_{i}^{q}$ is non-convex for $0<q<1$ and exhibits an increasing number of local optima as $q \rightarrow 0^{+}$. We present a heuristic polynomial time algorithm in the following.

Algorithm 1 Initialize $S_{0}=S$ and $k=0$.

Step 1: Solve the linear program

$$
\begin{aligned}
& \left(X^{(k)}, \beta^{(k)}\right)=\arg \min _{X, \beta} \beta, \\
& L_{i}-\beta \leq a_{i}^{\top} X \leq U_{i}+\beta \\
& \beta \geq 0
\end{aligned}
$$

where $\left(a_{i}, L_{i}, U_{i}\right) \in S_{k}$.

Step 2: If $\beta^{(k)}=0$ (or smaller than a predetermined positive threshold) then propose $X^{(k)}$ and $S_{k}$ as the solution and terminate. Otherwise continue.

Step 3: Determine all the elements of $S_{k}$ that are involved in at least one of the $p+1$ active constraints of the linear program (2.1). These elements constitute candidates for removal from the set (ie there is a maximum number of $p+1$ elements in the candidate set at each iteration).

Step 4: Select one element of the candidate set for removal from $S_{k}$. Update $k$ to $k+1$ and go to Step 1.
The algorithm may be explained as follows. At each iteration, say iteration $k$, the linear program (2.1) minimizes the maximum positive deviation from the bounds for the inequalities induced by $S_{k}$, or more precisely, minimizes $\max \left\{0, \max _{i}\left\{X^{\top} a_{i}-U_{i}, L_{i}-X^{\top} a_{i}\right\}\right\}$ for $\left(a_{i}, L_{i}, U_{i}\right) \in S_{k}$. The constraints of the linear program (2.1) are obviously feasible (since $\beta$ should merely be selected large enough for the inequalities to hold) and the feasibility of the subsystem $L_{i} \leq X^{\top} a_{i} \leq U_{i}$, $\left(a_{i}, L_{i}, U_{i}\right) \in S_{k}$, holds iff $\beta^{(k)}=0$ (the termination criterion of Step 2) . The algorithm then proceeds by removing an element from $S_{k}$ that results in a small value for $\beta^{(k+1)}$. Removal of an element that is not a candidate proposed by Step 3 will obviously leave the optimal value of $\beta^{(k+1)}$ unchanged relative to $\beta^{(k)}$. Hence, we only consider removal of $S_{k}$ 's elements that are contained in the candidate set (Step 4). One way of selecting among candidate elements is removal of each candidate at a time, solving the linear program followed by the removal, and selecting the element whose removal results in the smallest value for $\beta^{(k+1)}$. Note that this approach is optimal in a one step ahead sense meaning that if removal of only one element renders a feasible subset then that element will be identified by the procedure. Note that this technique requires solutions to (at most) $p+1$ linear programs at each iteration. Other techniques might be proposed to speed up calculations, eg removal of the element involved in an equality with largest Lagrange multiplier value at the solution to $(2.1)$

\section{Numerical Examples}

In this section, we present two numerical examples to illustrate the performance of the algorithm. The first one is a simulated case where it is of interest to maximize the number of feasible two sided bounds. The second example is an application from the area of radiotherapy planning.

A two sided case: Consider the system of two sided inequalities $19.5 \leq a_{i}^{\top} X \leq 20.5, i=1, \cdots, 100$, where the elements of $a_{i} \in \mathcal{R}^{20}$ are randomly sampled from a uniform distribution between 0.75 and 1.25. Denoting $\alpha_{i}=\max \left\{0, \max \left\{X^{\top} a_{i}-U_{i}, L_{i}-X^{\top} a_{i}\right\}\right\}$, we apply Algorithm 1 to minimize $\sum_{i} \alpha_{i}^{0^{+}}$where the removal is optimal in a one step ahead sense. The percentage of satisfied constraints upon termination of the algorithm is $88 \%$ and the computations take about $3 \frac{3}{4}$ minutes on a HP-PA 8000 work station using MATLAB 5.2 software. Further, we apply linear programming to minimize $\sum_{i} \alpha_{i}$ as a convex approximation to the original problem. The percentage of satisfied constraints for 
the solution of the linear program is $74 \%$ which is noticeably lower than the result obtained by Algorithm 1.

Radiotherapy planning: Coronary angioplasty is a minimally invasive technique for treatment of atherosclerosis, the principle process of heart disease. Despite its wide acceptance, coronary angioplasty is limited by rates of restenosis (renarrowing of the vessel) of 30-60\% [9]. A major component of the restenosis process is intimal hyperplasia which refers to the proliferative response to vascular injury. While coronary stents virtually remove recoil and remodeling (other components of the restenosis process) they do not decrease the proliferative response caused by angioplasty. Recent studies show that ionizing radiation, administered during or after angioplasty, can inhibit the proliferative component of restenosis [10]. Possible radiation techniques are temporary or high dose rate catheter-based brachytherapy using radioactive seeds, wire, or liquid filled balloons, permanent or low dose rate brachytherapy using radioactive stents, and external beam radiation. Treatment planning is essential since low doses of radiation stimulate neointimal proliferation and high doses may cause vascular complications [11].

The first step in the design of an optimal treatment plan is outlining points on tomographic sections of the vessel (obtained by eg ultrasonography) that should receive proper amounts of radioactive dose. These points are typically selected on an inner and an outer surface that circumscribe a smooth muscle cell layer of the vessel [9]. The next step is determination of the treatment variables so as to deliver desirable dose values to the outlined points. In this example, we consider the common technique of high dose rate radiation delivery where an afterloader is used to step a train of seeds along the vessel path. The train of seeds dwells at predetermined positions (dwell positions) along the path for predetermined durations of time (dwell times). The treatment variable consists of delivery configuration parameters (relative positioning of the seeds within a train, initial and final positions of the trains on the paths, stepping lengths, seed geometry, etc.) and the sequence of dwell times. Here we consider dwell time optimization for a fixed delivery configuration. With the assumption that the total irradiation times are much smaller than seeds half lives, the dose delivery rates may be assumed constant within the time intervals of radiation. Then for a train of seeds dwelling at a certain position along its path, the amount of delivered dose to a point is equal to the dwell time of the train multiplied by the dose delivery rate to the point of interest for that dwell position. Moreover, the dose delivered during transition between consequent dwell positions may be ignored due to fast afterloading (negligible transition times). Hence, the dose delivered to a point, say $v$, is given by $\sum_{i=1}^{p} \phi_{i}(v) T_{i}$ where $\phi_{i}(v)$ is dose delivery rate at point $v$ from the dwell position $i, T_{i}$ is the dwell time $i$, and $p$ is the total number of dwell positions. Since the dose delivery rate to a point is only affected by the delivery configuration (which is assumed to be fixed), the problem of treatment planning concerns optimal selection of $T=\left(T_{1}, \cdots, T_{p}\right)^{\top}$. The desirable dose values are most commonly given as bounds on delivered radioactive dose to the outlined points. The ideal dose bounds for treatment of intimal hyperplasia are usually given as maximum $D_{\max }=30$ Gy (radiation unit) for the points on the inner surface and minimum $D_{\min }=8 \mathrm{~Gy}$ for the points on the outer surface [9]. These objectives may be stated as

$$
\begin{aligned}
& \sum_{i=1}^{p} \phi_{i}(v) T_{i} \leq D_{\max } \forall v \in \text { inner surface, } \\
& \sum_{i=1}^{p} \phi_{i}(v) T_{i} \geq D_{\min } \forall v \in \text { outer surface. }
\end{aligned}
$$

Under infeasibility of the above prescribed bounds, an alternative will be maximization of the fraction of outlined points that receive dose values within the prescribed bounds, ie maximum feasible subset problem for the system (3.2). Indeed, numbers such as fraction of a volume or surface receiving desirable dose values are commonly used in clinical practice for assessment of a treatment plan and are closely related to the so called dose-volume histograms (DVH) or dose-surface histograms (DSH). DVH's and DSH's are cumulative versions of histogram over delivered dose to the volume or surface of interest and are widely accepted figures of merit for evaluation of a treatment plan [12].

Optimization results are presented for a vessel with tubular inner and outer surfaces where the inner surface narrows in the middle of the vessel (see Figure 1) and the delivery configuration and point sampling are identical to those of the numerical study of [13]. The point sampling and delivery configuration used here result in a system of 288 inequalities and 6 variables (ie $N=288$ and $p=6$ ). Similar to [13], calculation of dose rates are based on line source approximation following the American Association of Physicists in Medicine (AAPM) task group 43 (TG43) formalism [14] as recommended by the AAPM-TG60 [15] for photon emitting sources.

Denoting $\alpha_{i}=\max \left\{0, \max \left\{X^{\top} a_{i}-U_{i}, L_{i}-X^{\top} a_{i}\right\}\right\}$, we apply Algorithm 1 to minimize $\sum_{i} \alpha_{i}^{0^{+}}$where the removal is optimal in a one step ahead sense. The percentage of satisfied constraints upon termination of the 


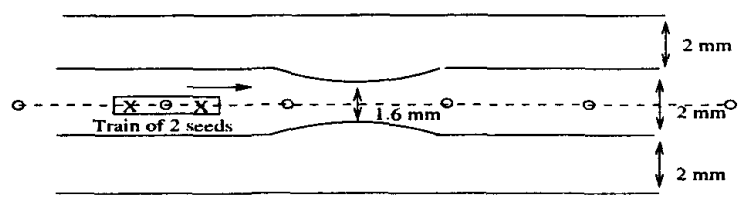

Figure 1: Longitudinal cross section of the vessel under radiation delivery. Seeds (marked by $X$ ) travel in a train of two (represented by the box containing the $\mathrm{X}$ marks). The train of seeds travels along the center line of the vessel (represented by the dashed line) and dwells at the positions marked by o along its path.

algorithm is $70 \%$ and the computations take about $7 \frac{2}{3}$ minutes on a HP-PA 8000 work station using MATLAB 5.2 software. Further, we apply linear programming to minimize $\sum_{i} \alpha_{i}$ as a convex approximation to the original problem. The percentage of satisfied constraints for the solution of the linear program is $62 \%$ which is again noticeably lower than the result obtained by Algorithm 1.

Finally, it should be noted that the planning problems arising in connection with radiotherapy of cancer are very similar to the planning problem considered here. The approach is therefore expected to be of enormous importance in radiotherapy of cancer.

\section{Conclusion}

We have presented a heuristic polynomial time algorithm for maximum feasible subset selection. The performance of the algorithm has been illustrated on a simulated linear system with double sided bounds and a case from radiation therapy planning for treatment of intimal hyperplasia. It will be of interest to test the performance of the algorithm versus existing other techniques and apply the maximum feasible subset approach to various treatment planning problems, eg radiotherapy of cancer.

\section{References}

[1] R. E. Warmack and R. C. Gonzalez. An algorithm for the optimal solution of linear inequalities and its application to pattern recognition. IEEE Transactions on Computers, C-22(12):1065-1075, 1973.

[2] S. I. Gallant. Perceptron-based learning algorithms. IEEE Transaction on Neural Networks, 1(2):179-191, 1990.

[3] M. Marchand and M. Golea. On learning simple neural concepts: from halfspace intersections to neural decision lists. Network: Computation in Neural Systems, 4(1):67-85, 1993.

[4] E. Amaldi and V. Kann. The complexity and approximability of finding maximum feasible subsystems of linear relations. Theoretical Computer Science, 147(1-2):181-120, 1995.

[5] J. W. Chinneck. An effective polynomial-time heuristic for the minimum cardinality IIS set-covering problem. Annals of Mathematics and Artificial Intelligence, 17:127-144, 1996.

[6] M. Parker and J. Ryan. Finding the minimum weight IIS cover of an infeasible system of linear inequalities. Annals of Mathematics and Artificial Intelligence, 17:107-126, 1996.

[7] J. W. Chinneck. Improved linear classification via LP infeasibility analysis. Technical Report SCE-9809, Department of Systems and Computer Engineering, Carleton University, Ottawa, Ontario, Canada, 1998.

[8] G. Brown and G. Graves. Elastic programming: a new approach to large-scale mixed integer optimization. Presented at ORSA/TIMS Conference, Las Vegas, 1975.

[9] P. S. Teirstein, V. Massullo, and S. Jani. Cathether-based radiotherapy to inhibit restenosis after coronary stenting. The New England Journal of Medicine, 336(24):1697-1703, June 1997.

[10] R. Waksman. Radiation for prevention of restenosis: where are we? Int. J. Radiat. Oncol. Biol. Phys., 36:959-961, 1996.

[11] D. A. Diamond and T. A. Vesely. The role of radiation therapy in the management of vascular restenosis. Part II. Radiation techniques and results. Journal of Vascular and Interventional Radiology, 9(3):389-400, 1998.

[12] S. Carlier, J. Marijnissen, and V. Coen. Guidance of intracoronary radiation therapy based on dosevolume histograms derived from quantitative intravascular ultrasound. IEEE Transactions on Medical Imaging, 17(5):772-778, 1998.

[13] P. Sadegh, F. A. Mourtada, R. H. Taylor, and J. H. Anderson. Brachytherapy optimal planning with application to intravascular radiation therapy. Submitted to Medical Image Analysis, 1999.

[14] R. Nath, L. L. Anderson, G. Luxton, K. A. Weaver, J. F. Williamson, and A. S. Meigooni. Dosimetry of interstitial brachytherapy sources: recommendations of the AAPM radiation therapy committee task group no. 43. Medical Physics, 22(2), February 1995.

[15] R. Nath and L. Liu. On the depth of penetration of photons and electrons for intravascular brachytherapy. Medical Physics, 24:1358, 1997. Abstract. 